\title{
Gauge transformations in the Lagrangian and Hamiltonian formalisms of generally covariant theories
}

\author{
J. M. Pons* \\ Departament d'Estructura i Constituents de la Matèria, Universitat de Barcelona, Avinguda Diagonal 647, \\ 08028 Barcelona, Catalonia, Spain \\ D. C. Salisbury ${ }^{\dagger}$ \\ Austin College, Sherman, Texas 75090 \\ L. C. Shepley \\ Center for Relativity, The University of Texas, Austin, Texas 78712-1081
}

(Received 29 July 1996)

\begin{abstract}
We study spacetime diffeomorphisms in the Hamiltonian and Lagrangian formalisms of generally covariant systems. We show that the gauge group for such a system is characterized by having generators which are projectable under the Legendre map. The gauge group is found to be much larger than the original group of spacetime diffeomorphisms, since its generators must depend on the lapse function and shift vector of the spacetime metric in a given coordinate patch. Our results are generalizations of earlier results by Salisbury and Sundermeyer. They arise in a natural way from using the requirement of equivalence between Lagrangian and Hamiltonian formulations of the system, and they are new in that the symmetries are realized on the full set of phase space variables. The generators are displayed explicitly and are applied to the relativistic string and to general relativity. [S0556-2821(97)06202-4]

PACS number(s): 04.20.Fy, 04.20.Cv
\end{abstract}

\section{INTRODUCTION}

The goal of this paper is to understand diffeomorphism symmetries in the canonical formalism at the classical level. The putative generators of infinitesimal general coordinate transformations feature in all canonical quantization approaches, but debate persists in the literature as to what aspects of the diffeomorphism group are realized at the classical level as canonical transformations [1-4]. This issue is intimately related to the meaning of time in quantum gravity.

In this paper we extend recent work by Pons and Shepley [5] concerning constrained systems. We analyze diffeomorphism symmetries using in a natural way the equivalence of the Hamiltonian and the Lagrangian approaches to generally covariant systems. We show that infinitesimal transformations which are projectable under the Legendre map are a basis for the generators of the gauge group. This group is much larger than the original group of spacetime diffeomorphisms because it acts on the space of spacetime metrics, whereas the diffeomorphism group acts on the underlying manifold. Since we retain the full set of canonical variables, the associated infinitesimal generators are new; they are realized on the full set of phase space variables and must at least depend in a specific way on the lapse function and shift vector of the spacetime metric in a given coordinate patch. The results are contrasted and compared with earlier work by Salisbury and Sundermeyer [4] on the realizability of general coordinate transformations as canonical transformations.

\footnotetext{
*Electronic address: pons@ecm.ub.es

${ }^{\dagger}$ Electronic address: dsalis@ austinc.edu

‡Electronic address: larry@ helmholtz.ph.utexas.edu
}

The formalism we shall develop encompasses all generally covariant Lagrangian dynamical models containing configuration variables which are either metric components or which may be used to construct a metric. We begin in Sec. II with a rederivation of the relation between gauge symmetries in Lagrangian and Hamiltonian formalisms. After introducing the notions of lapse and shift in Sec. III, we show that diffeomorphism-induced gauge transformations are projectable under the Legendre transformation if and only if infinitesimal variations depend on the lapse and shift but not on their time derivatives. These projectable infinitesimal transformations thus contain a compulsory dependence on the normal to the chosen time foliation. We illustrate these ideas with the relativistic particle, canonical gravity, and the relativistic string.

In Sec. IV we turn our attention to the construction of canonical generators of the metric-dependent gauge group. These objects generate symmetry transformations on the full set of canonical variables. We show that every generator with nonvanishing time component acts as an evolution generator on at least one member of every equivalence class of solutions. Section V contains a discussion of gauge fixing and the elimination of redundancy in initial conditions. In Sec. VI, our conclusion, we discuss the nature of the diffeomorphism-induced gauge group. The Appendix illustrates the projectability conditions in a model, the NambuGoto string, in which the lapse and shift depend on time derivatives of the dynamical variables.

\section{NOETHER HAMILTONIAN SYMMETRIES}

We begin by rederiving some results of Batlle et al. [6] for first order Lagrangians $L(q, \dot{q})$. We exclude Lagrangians 
which explicitly depend on time $t$ since we are interested in reparametrization covariant systems. We start with a Noether Lagrangian symmetry

$$
\delta L=d F / d t
$$

and we will investigate the conversion of this symmetry to the Hamiltonian formalism. Defining

$$
G=\left(\partial L / \partial \dot{q}^{i}\right) \delta q^{i}-F
$$

we can write

$$
[L]_{i} \delta q^{i}+\frac{d G}{d t}=0
$$

where $[L]_{i}$ is the Euler-Lagrange functional derivative of $L$,

$$
[L]_{i}=\alpha_{i}-W_{i s} \ddot{q}^{s},
$$

where

$$
W_{i j} \equiv \frac{\partial^{2} L}{\partial \dot{q}^{i} \partial \dot{q}^{j}} \quad \text { and } \quad \alpha_{i} \equiv-\frac{\partial^{2} L}{\partial \dot{q}^{i} \partial q^{s}} \dot{q}^{s}+\frac{\partial L}{\partial q^{i}} .
$$

Here we consider the general case where the mass matrix or Hessian $\mathbf{W}=\left(W_{i j}\right)$ may be a singular matrix. In this case there exists a kernel for the pullback $\mathcal{F} L^{*}$ of the Legendre map $\mathcal{F} L$ from configuration-velocity space $T Q$ (the tangent bundle $T Q$ of the configuration space $Q$ ) to phase space $T^{*} Q$ (the cotangent bundle). This kernel is spanned by the vector fields

$$
\boldsymbol{\Gamma}_{\mu}=\gamma_{\mu}^{i} \frac{\partial}{\partial \dot{q}^{i}},
$$

where $\gamma_{\mu}^{i}$ are a basis for the null vectors of $W_{i j}$. The Lagrangian time-evolution differential operator can therefore be expressed as

$\mathbf{X}=\frac{\partial}{\partial t}+\dot{q}^{s} \frac{\partial}{\partial q^{s}}+a^{s}(q, \dot{q}) \frac{\partial}{\partial \dot{q}^{s}}+\lambda^{\mu} \boldsymbol{\Gamma}_{\mu} \equiv \mathbf{X}_{0}+\lambda^{\mu} \boldsymbol{\Gamma}_{\mu}$,

where $a^{s}$ are functions which are determined by the formalism, and $\lambda^{\mu}$ are arbitrary functions. It is not necessary to use the Hamiltonian technique to find the $\boldsymbol{\Gamma}_{\mu}$, but it does facilitate the calculation:

$$
\gamma_{\mu}^{i}=\mathcal{F} L^{*}\left(\frac{\partial \phi_{\mu}}{\partial p_{i}}\right)
$$

where the $\phi_{\mu}$ are the Hamiltonian primary first class constraints.

Notice that the highest derivative in Eq. (2.2), $\ddot{q}^{i}$, appears linearly. Because $\delta L$ is a symmetry, Eq. (2.2) is identically satisfied, and therefore the coefficient of $\ddot{q}^{i}$ vanishes:

$$
W_{i s} \delta q^{s}-\frac{\partial G}{\partial \dot{q}^{i}}=0
$$

We contract with a null vector $\gamma_{\mu}^{i}$ to find that

$$
\Gamma_{\mu} G=0 .
$$

It follows that $G$ is projectable to a function $G_{\mathrm{H}}$ in $T^{*} Q$; that is, it is the pullback of a function (not necessarily unique) in $T^{*} Q$ :

$$
G=\mathcal{F} L^{*}\left(G_{\mathrm{H}}\right) .
$$

This important property, valid for any conserved quantity associated with a Noether symmetry, was first pointed out by Kamimura [7]. Observe that $G_{\mathrm{H}}$ is determined up to the addition of linear combinations of the primary constraints. Substitution of this result in Eq. (2.6) gives

$$
W_{i s}\left[\delta q^{s}-\mathcal{F} L^{*}\left(\frac{\partial G_{\mathrm{H}}}{\partial p_{s}}\right)\right]=0,
$$

and so the brackets enclose a null vector of $\mathbf{W}$ :

$$
\delta q^{i}-\mathcal{F} L^{*}\left(\frac{\partial G_{\mathrm{H}}}{\partial p_{i}}\right)=\sum_{\mu} r^{\mu} \gamma_{\mu}^{i},
$$

for some $r^{\mu}(t, q, \dot{q})$.

We shall investigate the projectability of variations generated by diffeomorphisms in the following section. Assume for now that an infinitesimal transformation $\delta q^{i}$ is projectable:

$$
\boldsymbol{\Gamma}_{\mu} \delta q^{i}=0
$$

Notice that if $\delta q^{i}$ is projectable, so must be $r^{\mu}$, so that $r^{\mu}=\mathcal{F} L^{*}\left(r_{\mathrm{H}}^{\mu}\right)$. Then, using Eqs. (2.5) and (2.7), we see that

$$
\delta q^{i}=\mathcal{F} L^{*}\left(\frac{\partial\left(G_{H}+\Sigma_{\mu} r_{\mathrm{H}}^{\mu} \phi_{\mu}\right)}{\partial p_{i}}\right) .
$$

We now redefine $G_{\mathrm{H}}$ to absorb the piece $\Sigma_{\mu} r_{\mathrm{H}}^{\mu} \phi_{\mu}$, and from now on we will have

$$
\delta q^{i}=\mathcal{F} L^{*}\left(\frac{\partial G_{\mathrm{H}}}{\partial p_{i}}\right) .
$$

Define

$$
\hat{p}_{i}=\frac{\partial L}{\partial \dot{q}^{i}} ;
$$

after eliminating Eq. (2.6) times $\ddot{q}^{i}$ from Eq. (2.2), we obtain

$$
\begin{aligned}
& \left(\frac{\partial L}{\partial q^{i}}-\dot{q}^{s} \frac{\partial \hat{p}_{i}}{\partial q^{s}}\right) \mathcal{F} L^{*}\left(\frac{\partial G_{\mathrm{H}}}{\partial p_{i}}\right)+\dot{q}^{i} \frac{\partial}{\partial q^{i}} \mathcal{F} L^{*}\left(G_{\mathrm{H}}\right)+\mathcal{F} L^{*}\left(\frac{\partial G_{\mathrm{H}}}{\partial t}\right) \\
& \quad=0
\end{aligned}
$$

which simplifies to

$$
\frac{\partial L}{\partial q^{i}} \mathcal{F} L^{*}\left(\frac{\partial G_{\mathrm{H}}}{\partial p_{i}}\right)+\dot{q}^{i} \mathcal{F} L^{*}\left(\frac{\partial G_{\mathrm{H}}}{\partial q^{i}}\right)+\mathcal{F} L^{*}\left(\frac{\partial G_{\mathrm{H}}}{\partial t}\right)=0 .
$$


Now let us invoke two identities [8] that are at the core of the connection between the Lagrangian and the Hamiltonian equations of motion. They are

$$
\dot{q}^{i}=\mathcal{F} L^{*}\left(\frac{\partial H}{\partial p_{i}}\right)+v^{\mu}(q, \dot{q}) \mathcal{F} L^{*}\left(\frac{\partial \phi_{\mu}}{\partial p_{i}}\right)
$$

and

$$
\frac{\partial L}{\partial q^{i}}=-\mathcal{F} L^{*}\left(\frac{\partial H}{\partial q^{i}}\right)-v^{\mu}(q, \dot{q}) \mathcal{F} L^{*}\left(\frac{\partial \phi_{\mu}}{\partial q^{i}}\right) ;
$$

where $H$ is any canonical Hamiltonian, so that $\mathcal{F} L^{*}(H)=\dot{q}^{i}\left(\partial L / \partial \dot{q}^{i}\right)-L=\hat{E}$, the Lagrangian energy, and the functions $v^{\mu}$ are determined so as to render the first relation an identity. Notice the important relation

$$
\boldsymbol{\Gamma}_{\mu} v^{\nu}=\delta_{\mu}^{\nu}
$$

which stems from applying $\boldsymbol{\Gamma}_{\mu}$ to the first identity and taking into account that

$$
\Gamma_{\mu} \circ \mathcal{F} L^{*}=0 .
$$

Substitution of these two identities into Eq. (2.9) yields (where $\{$,$\} is the Poisson bracket)$

$$
\mathcal{F} L^{*}\left\{G_{\mathrm{H}}, H\right\}+v^{\mu} \mathcal{F} L^{*}\left\{G_{\mathrm{H}}, \phi_{\mu}\right\}+\mathcal{F} L^{*}\left(\frac{\partial G_{\mathrm{H}}}{\partial t}\right)=0 .
$$

This result can be split through the action of $\boldsymbol{\Gamma}_{\mu}$ into

$$
\mathcal{F} L^{*}\left\{G_{\mathrm{H}}, H\right\}+\mathcal{F} L^{*}\left(\frac{\partial G_{\mathrm{H}}}{\partial t}\right)=0
$$

and

$$
\mathcal{F} L^{*}\left\{G_{\mathrm{H}}, \phi_{\mu}\right\}=0
$$

or, equivalently,

$$
\left\{G_{\mathrm{H}}, H\right\}+\left(\frac{\partial G_{\mathrm{H}}}{\partial t}\right)=p c
$$

and

$$
\left\{G_{\mathrm{H}}, \phi_{\mu}\right\}=p c,
$$

where $p c$ stands for any linear combination of primary constraints. We have arrived at a neat characterization for a generator $G_{\mathrm{H}}$ of Noether transformations in the canonical formalism.

Up to now we have considered general Noether symmetries, encompassing rigid (global) as well as gauge (local) transformations. Let us finally specialize to gauge transformations. For reparametrization covariant theories, except for a small number of exceptional cases not important for this paper [9], a gauge generator will be of the form

$$
G_{\mathrm{H}}(t)=\epsilon(t) G_{0}(q, p)+\dot{\epsilon}(t) G_{1}(q, p),
$$

where $\epsilon(t)$ is an arbitrary function. Because of the arbitrariness of $\epsilon(t)$, and recognizing that the Poisson brackets of the
Hamiltonian with primary constraints yields secondary constraints, we learn from Eq. (2.13) that

$$
\begin{gathered}
G_{1}=p c, \\
G_{0}=-\left\{G_{1}, H\right\}+p c,
\end{gathered}
$$

and

$$
\left\{G_{0}, H\right\}=p c
$$

while from Eq. (2.14) we deduce that

$$
\left\{G_{0}, p c\right\}=p c
$$

and

$$
\left\{G_{1}, p c\right\}=p c .
$$

It can be shown from Eq. (2.15) that $G_{0}$ must contain a piece which is a secondary constraint, while Eq. (2.17) and Eq. (2.18) show that both $G_{0}$ and the primary constraint $G_{1}$ are first class.

\section{DIFFEOMORPHISM-INDUCED GAUGE SYMMETRIES}

We specialize now to generally covariant dynamical models in which a metric can be constructed with the configuration variables (but not with velocity variables). We assume in addition that no further gauge symmetry exists. We shall illustrate our results with the relativistic particle with an auxiliary variable and with general relativity. Our first objective is to determine the general form of projectable variations resulting from diffeomorphisms on a coordinate patch.

If a metric exists in a coordinate system $\left\{x^{\mu}\right\}$ the line element may always be written in the form

$$
d s^{2}=-N^{2}\left(d x^{0}\right)^{2}+g_{a b}\left(N^{a} d x^{0}+d x^{a}\right)\left(N^{b} d x^{0}+d x^{b}\right)
$$

with contravariant metric components given by

$$
\left(g^{\mu \nu}\right)=\left(\begin{array}{cc}
-N^{-2} & N^{-2} N^{a} \\
N^{-2} N^{a} & e^{a b}-N^{-2} N^{a} N^{b}
\end{array}\right),
$$

with $e^{a b} g_{b c}=\delta_{c}^{a}$. The lapse function $N$ and shift vector $N^{a}$ will play important roles in our discussion. Our index conventions are that greek indices range from 0 to $M$, where $M$ is the dimension of the spacelike hypersurfaces of the time foliation. Latin indices range from 1 to $M$.

Explicitly, the configuration space variables are $N^{\mu}$ (with $N^{0} \equiv N$ ) and $g_{a b}$. The unit normal $n^{\mu}$ to the spacelike hypersurfaces is given by

$$
n^{\mu}=\delta_{0}^{\mu} N^{-1}-\delta_{a}^{\mu} N^{-1} N^{a} \text {, so that } n^{\mu} n^{\nu} g_{\mu \nu}=-1 .
$$

Since $e^{a b}$ is the inverse of the three-metric $g_{a b}$, the contravariant components of the spacetime metric are

$$
g^{\mu \nu}=e^{a b} \delta_{a}^{\mu} \delta_{b}^{\nu}-n^{\mu} n^{\nu}
$$

Diffeomorphism covariance prevents the lapse $N$ and shift $N^{a}$ from being fixed by the equations of motion in any generally covariant dynamical model. Specifically, since the 
$N^{\mu}$ are arbitrary, $\ddot{N}^{\mu}$ are undetermined. The evolution operator (2.4) acting on $\dot{N}^{\mu}$ must therefore serve only to relate the arbitrary functions $\lambda^{\mu}$ to the $\ddot{N}^{\mu}$. Consequently, $\boldsymbol{\Gamma}_{\mu} \dot{N}^{\nu}$ must form a nonsingular matrix. Further, $\boldsymbol{\Gamma}_{\mu}$ acting on any other velocity must give zero, since we are assuming no other gauge symmetry. It follows that the null vectors of the Hessian $\mathbf{W}$ [see Eq. (2.3)] are spanned by

$$
\boldsymbol{\Gamma}_{\mu}=\frac{\partial}{\partial \dot{N}^{\mu}} .
$$

Since there are $M+1$ of the $N^{\mu}$, these null vectors span the arbitrary component of the Lagrangian evolution operator (2.4).

Now consider infinitesimal coordinate transformations $x^{\mu} \rightarrow x^{\mu}-\epsilon^{\mu}(x)$, with $\epsilon^{\mu}$ arbitrary functions of the coordinate variables $x^{\nu}$. The corresponding variations of the components of the metric tensor (the Lie derivative of the metric along $\left.\epsilon^{\mu}\right)$ are $\left({ }_{, \mu} \equiv \partial / \partial x^{\mu}\right)$

$$
\delta g^{\mu \nu}=g_{, \rho}^{\mu \nu} \epsilon^{\rho}-g^{\mu \rho} \epsilon_{, \rho}^{\nu}-g^{\rho \nu} \epsilon_{, \rho}^{\mu},
$$

or

$$
\delta g_{\mu \nu}=g_{\mu \nu, \rho} \epsilon^{\rho}+g_{\mu \rho} \epsilon_{, \nu}^{\rho}+g_{\rho \nu} \epsilon_{, \mu}^{\rho} .
$$

The variations of the $N^{\mu}$ are readily calculated:

$$
\begin{gathered}
\delta N=N_{, \mu} \epsilon^{\mu}+N \epsilon_{, 0}^{0}-N N^{a} \epsilon_{, a}^{0}, \\
\delta N^{a}=N_{, \mu}^{a} \epsilon^{\mu}+N^{a} \epsilon_{, 0}^{0}-\left(N^{2} e^{a b}+N^{a} N^{b}\right) \epsilon_{, b}^{0}+\epsilon_{, 0}^{a}-N^{b} \epsilon_{, b}^{a} .
\end{gathered}
$$

Thus the variations of the $N^{\mu}$ do depend on $\dot{N}^{\mu}=N_{.0}^{\mu}$ (but the variations of $g_{a b}$ do not), assuming as we have above, that $\epsilon^{\mu}$ depends only on the coordinates. Consequently the variations of $N^{\mu}$ are clearly not projectable; projectability is attained only if we permit $\epsilon^{\mu}$ to depend on $N^{\mu}$. The requirement that derivatives of $\delta N^{\mu}$ with respect to $\dot{N}^{\mu}$ vanish implies that

$$
\begin{gathered}
\epsilon^{0}+N \frac{\partial \epsilon^{0}}{\partial N}=0, \\
\frac{\partial \epsilon^{0}}{\partial N^{a}}=0, \\
N^{a} \frac{\partial \epsilon^{0}}{\partial N}+\frac{\partial \epsilon^{a}}{\partial N}=0, \\
\epsilon^{0} \delta_{b}^{a}+\frac{\partial \epsilon^{a}}{\partial N^{b}}=0 .
\end{gathered}
$$

These equations were first obtained in [4] using a rather different approach. In [4], the following requirement was introduced for diffeomorphism-induced gauge transformations: Consider $\delta_{1} x^{\mu}=-\epsilon_{1}^{\mu}(x, g(x))$ and $\delta_{2} x^{\mu}=-\epsilon_{2}^{\mu}(x, g(x))$; then ask for conditions to be satisfied by $\epsilon_{1}, \epsilon_{2}$ such that $\left[\delta_{1}, \delta_{2}\right] x^{\mu}$ has no explicit time derivatives of $\epsilon_{1}$ or $\epsilon_{2}$. We will discuss in the next section the reason why this latter approach gives results coincident with ours. We feel that the requirement of projectability (independence of the $\delta N^{\mu}$ on $\dot{N}^{\mu}$ in this case) is a more natural approach.

The general solution of the $\epsilon^{\mu}$ equations (3.10)-(3.13) is

$$
\epsilon^{\mu}=\delta_{a}^{\mu} \xi^{a}+n^{\mu} \xi^{0}, \text { so that } \epsilon^{0}=\frac{\xi^{0}}{N}, \epsilon^{a}=\xi^{a}-\frac{N^{a}}{N} \xi^{0},
$$

where $\xi^{a}$ and $\xi^{0}$ are arbitrary functions of the spacetime variables $x^{\mu}$ and $g_{a b}$ but are independent of $N^{\mu}$. The dependence on the $M$-surface metric plays no role in our present arguments but is required, as we show in Sec. VI, in order that the diffeomorphism-induced transformations form a group. The result (3.14) is true in a more general context than we have been treating. The Appendix will illustrate this point with an example, the Nambu-Goto string, in which the metric is built with velocity variables as well as configuration space variables.

\section{A. Free relativistic particle with auxiliary variable}

We illustrate first with the unit-mass relativistic free particle model with auxiliary variable described by the Lagrangian

$$
L=\frac{1}{2 e} \dot{x}^{\mu} \dot{x}^{\nu} \eta_{\mu \nu}-\frac{1}{2} e,
$$

where $x^{\mu}$ is the vector variable in Minkowski spacetime, with metric $\left(\eta_{\mu \nu}\right)=\operatorname{diag}(-1,1,1,1)$, and $e$ is an auxiliary variable whose equation of motion gives $e=\left(-\dot{x}^{\mu} \dot{x}_{\mu}\right)^{1 / 2}$. Substituting this value of $e$ into the Lagrangian leads to the free particle Lagrangian $-\left(-\dot{x}^{\mu} \dot{x}_{\mu}\right)^{1 / 2}$.

The following Noether gauge transformation is well known to describe the reparametrization invariance for this Lagrangian $[\delta L=(d / d t)(\epsilon L)]$ :

$$
\delta x^{\mu}=\epsilon \dot{x}_{\mu}, \quad \delta e=\epsilon \dot{e}+\dot{\epsilon} e .
$$

Here $\epsilon$ is an infinitesimal arbitrary function of the evolution parameter $t$. Comparing Eq. (3.16) with Eq. (3.8), we observe that $e$ may be interpreted as a lapse, with corresponding metric $g_{00}=-e^{2}$.

The kernel of the pullback map $\mathcal{F} L^{*}$ is defined in Eq. (2.3); here it is spanned by the vector field $\boldsymbol{\Gamma}=\partial / \partial \dot{e}$. The condition that a function $f$ in configuration-velocity space be projectable to phase space is

$$
\boldsymbol{\Gamma} f=\frac{\partial f}{\partial \dot{e}}=0 .
$$

The Noether transformation (3.16) is not projectable to phase space, since $\boldsymbol{\Gamma} \delta e \neq 0$. Projectable transformations are of the form (3.14):

$$
\epsilon(t, e)=\xi(t) / e .
$$

The Noether variations then become 


$$
\delta x^{\mu}=\xi \frac{\dot{x}^{\mu}}{e}, \quad \delta e=\dot{\xi}
$$

The arbitrary function describing the Noether gauge transformation is $\xi(t)$. What we have achieved is a change of the generator of the gauge transformations. This leads to a change of the gauge algebra which in our case becomes Abelian. But from the point of view of the gauge symmetry of our model we still have the same mappings of solutions onto gauge equivalent solutions. That is, on a given dynamical trajectory $x_{0}^{\mu}(t), e_{0}(t)$ we can match the transformation given by an arbitrary $\epsilon_{0}(t)$ with $\xi_{0}(t)$ defined as $\xi_{0}(t) \equiv e_{0}(t) \epsilon_{0}(t)$. It is in comparing a transformation on one dynamical trajectory with that acting on another where the change has occurred. In Sec. VI we shall elaborate further on the issue of the gauge group.

The canonical Hamiltonian is

$$
H=\frac{1}{2} e\left(p^{\mu} p_{\mu}+1\right)
$$

and there is a primary constraint $\pi \simeq 0$, where $\pi$ is the variable conjugate to $e$. The evolution operator vector field $\{-, H\}+\lambda(t)\{-, \pi\}$ yields the secondary constraint $\frac{1}{2}\left(p^{\mu} p_{\mu}+1\right) \simeq 0$. Both the primary and the secondary constraints are first class. The arbitrary function $\lambda$ is a reflection of the gauge invariance of the model. The solutions of the equations of motion are

$$
\begin{gathered}
x^{\mu}(t)=x^{\mu}(0)+p^{\mu}(0)\left(e(0) t+\int_{0}^{t} d \tau \int_{0}^{\tau} d \tau^{\prime} \lambda\left(\tau^{\prime}\right)\right), \\
e(t)=e(0)+\int_{0}^{t} d \tau \lambda(\tau), \\
p^{\mu}(t)=p^{\mu}(0), \\
\pi(t)=\pi(0),
\end{gathered}
$$

with the initial conditions satisfying the constraints.

Gauge transformations relate trajectories obtained through different choices of $\lambda(t)$. Consider an infinitesimal change $\lambda \rightarrow \lambda+\delta \lambda$. Then the change in the trajectories (keeping the initial conditions intact) is

$$
\begin{gathered}
\delta x^{\mu}(t)=p^{\mu}(0)\left(\int_{0}^{t} d \tau \int_{0}^{\tau} d \tau^{\prime} \delta \lambda\left(\tau^{\prime}\right)\right), \\
\delta e(t)=\int_{0}^{t} d \tau \delta \lambda(\tau) \\
\delta p^{\mu}(t)=0, \quad \delta \pi(t)=0
\end{gathered}
$$

which is nothing but a particular case of the projectable gauge transformations displayed above with

$$
\xi(t)=\int_{0}^{t} d \tau \int_{0}^{\tau} d \tau^{\prime} \delta \lambda\left(\tau^{\prime}\right)
$$

\section{B. Diffeomorphisms in canonical general relativity}

Up to a boundary piece, the Einstein-Hilbert Lagrangian can be written as [10]

$$
\mathcal{L}=\left({ }^{3} g\right){ }^{1 / 2} N\left({ }^{3} R+K_{a b} K^{a b}-K^{2}\right),
$$

where ${ }^{3} g$ is the determinant of the three-metric tensor in Eq. (3.1), ${ }^{3} g=\operatorname{det}\left(g_{a b}\right),{ }^{3} R$ is the scalar curvature computed from the three-metric, and $K_{a b}$ is the second fundamental form (extrinsic curvature) for the constant-time threesurfaces:

$$
K_{a b}=\frac{1}{2 N}\left(\dot{g}_{a b}-N_{a \mid b}-N_{b \mid a}\right)
$$

with meaning covariant differentiation with respect to the three-metric connection. Notice that the lapse $N$ and shift $N^{a}$ of the four-metric all appear, but their time-derivatives do not.

We may directly apply our general formalism, with no notational changes, to conclude that projectable infinitesimal coordinate transformations must be of the form

$$
x^{\mu} \rightarrow x^{\mu}-\delta_{a}^{\mu} \xi^{a}-n^{\mu} \xi^{0} .
$$

Notice also that for any specific spacetime metric $g_{\mu \nu}(x)$ we can implement any infinitesimal diffeomorphism $x^{\mu} \rightarrow x^{\mu}-\epsilon^{\mu}(x)$ by taking the set $\xi^{0}, \xi^{i}$ (assuming $N \neq 0$ ) as

$$
\xi^{0}=N \epsilon^{0}, \quad \xi^{a}=\epsilon^{a}+N^{a} \epsilon^{0} ;
$$

therefore we are not restricting the (infinitesimal) diffeomorphisms that can act on any specific metric. What we achieved is a set of generators of the gauge group which can be projected to the phase space.

\section{HAMILTONIAN GAUGE GENERATORS}

Our objective in this section is to derive the full set of diffeomorphism-induced gauge generators for the class of dynamical models treated in Sec. II. Since the $\xi^{\mu}$ are now the arbitrary functions of time appearing in the variations $\delta q^{i}$ of Sec. II, we modify the argument leading to the general form of the symmetry generators to conclude that these generators must be of the form

$$
G(t)=\int d^{M_{x}}\left(\xi^{\mu}(\mathbf{x}, t) G_{\mu}^{(0)}+\xi^{\mu}(\mathbf{x}, t) G_{\mu}^{(1)}\right) .
$$

When we use $\epsilon^{\mu}=\delta_{a}^{\mu} \xi^{a}+n^{\mu} \xi^{0}$ in Eq. (3.6), the algebra of the infinitesimal transformations ceases to be the standard diffeomorphism algebra. The standard algebra is that of Lie derivatives: $\epsilon_{3}^{\mu}=\epsilon_{2}^{\nu} \epsilon_{1, \nu}^{\mu}-\epsilon_{1}^{\nu} \epsilon_{2, \nu}^{\mu}=\left(\mathcal{L}_{\epsilon_{2}} \epsilon_{1}\right)^{\mu}$. In our case the commutator of two infinitesimal transformations yields an $\epsilon_{3}^{\mu}$ of the form of Eq. (3.14), with

$$
\begin{gathered}
\xi_{3}^{a}=\xi_{2}^{b} \xi_{1, b}^{a}-\xi_{1}^{b} \xi_{2, b}^{a}-e^{a b} \xi_{1}^{0} \xi_{2, b}^{0}+e^{a b} \xi_{2}^{0} \xi_{1, b}^{0}, \\
\xi_{3}^{0}=\xi_{2}^{a} \xi_{1, a}^{0}-\xi_{1}^{a} \xi_{2, a}^{0} .
\end{gathered}
$$

These are the new commutation relations of the gauge algebra in configuration-velocity space. The commutation rules of the gauge generators in phase space coincide with 
the commutation relations in configuration-velocity space as long as at least all but one of the $M+1$ gauge generators are linear in the momenta (see [11]). This amount of linearity holds in our models: Eq. (3.14) implies that timeindependent $M$-space diffeomorphisms are always projectable. This means that each associated canonical generator must be linear in the momenta; otherwise the transformation of configuration variables will depend on velocities. We can conclude that we know the Poisson bracket rules $G\left[\xi_{3}\right]=\left\{G\left[\xi_{1}\right], G\left[\xi_{2}\right]\right\}$ for our gauge generators. Thus in comparing Eq. (4.1) and Eq. (4.2) with $G\left[\xi_{3}\right]$ we deduce the algebra for these generators. In what follows, we use the convention that repeated indices imply both summation and $M$-dimensional integration; we use primed indices where necessary to make sure that separate integrations are clearly delineated, though we drop the primes on the indices where no loss of clarity is involved:

$$
\begin{gathered}
\left\{G_{\mu}^{(0)}, G_{\nu^{\prime}}^{(0)}\right\}=C_{\mu \nu^{\prime}}^{\alpha^{\prime \prime}} G_{\alpha^{\prime \prime}}^{(0)}+\left(\frac{d}{d t} C_{\mu \nu^{\prime}}^{\alpha^{\prime \prime}}\right) G_{\alpha^{\prime \prime}}^{(1)}, \\
\left\{G_{\mu}^{(0)}, G_{\nu^{\prime}}^{(1)}\right\}=C_{\mu \nu^{\prime}}^{\alpha^{\prime \prime}} G_{\alpha^{\prime \prime}}^{(1)},
\end{gathered}
$$

and

$$
\left\{G_{\mu}^{(1)}, G_{\nu^{\prime}}^{(1)}\right\}=0,
$$

where the structure coefficients are given by

$$
\begin{gathered}
C_{00^{\prime}}^{a^{\prime \prime}}=e^{a b}\left(\mathbf{x}^{\prime \prime}\right)\left(\delta^{M}\left(\mathbf{x}-\mathbf{x}^{\prime \prime}\right)+\delta^{M}\left(\mathbf{x}^{\prime}-\mathbf{x}^{\prime \prime}\right)\right) \frac{\partial}{\partial x^{b}} \delta^{M}\left(\mathbf{x}-\mathbf{x}^{\prime}\right) \\
C_{00^{\prime}}^{0^{\prime \prime}}=0 \\
C_{a 0^{\prime}}^{0^{\prime \prime}}=\delta^{M}\left(\mathbf{x}-\mathbf{x}^{\prime \prime}\right) \frac{\partial}{\partial x^{a}} \delta^{M}\left(\mathbf{x}-\mathbf{x}^{\prime}\right)=-C_{0^{\prime} a}^{0^{\prime \prime}}, \\
C_{a 0^{\prime}}^{b^{\prime \prime}}=0 \\
C_{a b^{\prime}}^{c^{\prime \prime}}=\left(\delta_{a}^{c} \delta^{M}\left(\mathbf{x}^{\prime \prime}-\mathbf{x}^{\prime}\right) \frac{\partial}{\partial x^{b}}+\delta_{b}^{c} \delta^{M}\left(\mathbf{x}^{\prime \prime}-\mathbf{x}\right) \frac{\partial}{\partial x^{a}}\right) \delta^{M}\left(\mathbf{x}-\mathbf{x}^{\prime}\right),
\end{gathered}
$$

and

$$
C_{a b^{\prime}}^{0^{\prime \prime}}=0 .
$$

We now construct these generators explicitly. The canonical Hamiltonian (such that its pullback under the Legendre transformation gives the Lagrangian energy) for the class of models under discussion is

$$
H=N^{\mu} \mathcal{H}_{\mu},
$$

where the $\mathcal{H}_{\mu}$ are independent of $N^{\mu}$ and $P_{\mu}$, and the primary constraints are $P_{\mu}$, the canonical variables conjugate to $N^{\mu}$. The secondary constraints are $\dot{P}_{\mu}=\left\{P_{\mu}, H\right\}$ $=-\mathcal{H}_{\mu}$, and no more constraints appear. It was shown in [5] that the canonical Hamiltonian always takes the form (4.12). All constraints are required to be first class; the rea- son is that $\xi^{\mu}$ and $\dot{\xi}^{\mu}$ appear in Eq. (3.6), and so all constraints are required to build the spacetime gauge generators that our theory possesses.

The Dirac Hamiltonian $H_{\mathrm{D}}$ is constructed by the addition to $H$ of a linear combination (with arbitrary functions $\lambda^{\mu}$ ) of the primary constraints:

$$
H_{\mathrm{D}}=H+\lambda^{\mu} P_{\mu} .
$$

$G_{\mu}^{(1)}$ must be a primary constraint, so the simplest choice is $G_{\mu}^{(1)}=P_{\mu}$. It is now necessary to apply Eq. (2.15): $G_{\mu}^{(0)}=-\left\{G_{\mu}^{(1)}, H\right\}+p c$, implying

$$
G_{\mu}^{(0)}=\mathcal{H}_{\mu}+A_{\mu}^{\nu} P_{\nu} .
$$

From Eq. (4.3) we deduce that

$$
\left\{\mathcal{H}_{\mu}, \mathcal{H}_{\nu}\right\}=C_{\mu \nu}^{\sigma} \mathcal{H}_{\sigma}
$$

since $\left\{N^{\mu}, \mathcal{H}_{\nu}\right\}=\left\{P_{\mu}, \mathcal{H}_{\nu}\right\}=0$.

The $A_{\mu}^{\nu}$ are determined by applying condition (2.16) to $G_{\mu}^{(0)}$ :

$$
\begin{aligned}
p c=\left\{\mathcal{H}_{\mu}+A_{\mu}^{\nu} P_{\nu}, H\right\} & =N^{\nu}\left\{\mathcal{H}_{\mu}, \mathcal{H}_{\nu}\right\}+A_{\mu}^{\nu}\left\{P_{\nu}, H\right\} \\
& =N^{\nu} C_{\mu \nu}^{\sigma} \mathcal{H}_{\sigma}-A_{\mu}^{\nu} \mathcal{H}_{\nu},
\end{aligned}
$$

which implies

$$
A_{\mu}^{\nu}=N^{\rho} C_{\mu \rho}^{\nu}
$$

up to an irrelevant arbitrary linear combination of primary constraints that would add an ineffective piece to the gauge generator. (By ineffective we mean that the added piece is quadratic in the constraints.) We ignore this piece and take the simplest solutions available for $A_{\mu}^{\nu}$.

It is trivial to check the fulfillment of conditions (2.17), (2.18). By use of the Jacobi identity we find

$$
\begin{aligned}
0 \equiv & \left\{\mathcal{H}_{\alpha},\left\{\mathcal{H}_{\beta}, \mathcal{H}_{\gamma}\right\}\right\}+\left\{\mathcal{H}_{\beta},\left\{\mathcal{H}_{\gamma}, \mathcal{H}_{\alpha}\right\}\right\}+\left\{\mathcal{H}_{\gamma},\left\{\mathcal{H}_{\alpha}, \mathcal{H}_{\beta}\right\}\right\} \\
= & \left(C_{\beta \gamma}^{\rho} C_{\alpha \rho}^{\sigma}+C_{\gamma \alpha}^{\rho} C_{\beta \rho}^{\sigma}+C_{\alpha \beta}^{\rho} C_{\gamma \rho}^{\sigma}\right) \mathcal{H}_{\sigma}+\left\{\mathcal{H}_{\alpha}, C_{\beta \gamma}^{\rho}\right\} \mathcal{H}_{\rho} \\
& +\left\{\mathcal{H}_{\beta}, C_{\gamma \alpha}^{\rho}\right\} \mathcal{H}_{\rho}+\left\{\mathcal{H}_{\gamma}, C_{\alpha \beta}^{\rho}\right\} \mathcal{H}_{\rho},
\end{aligned}
$$

together with

$$
\frac{d}{d t} C_{\alpha \beta}^{\gamma}=N^{\rho}\left\{C_{\alpha \beta}^{\gamma}, \mathcal{H}_{\rho}\right\},
$$

and it is straightforward to show that the generators $G_{\mu}^{(0)}$ and $G_{\mu}^{(1)}$ do satisfy the algebra (4.3)-(4.5).

We have therefore obtained the full set of diffeomorphism-induced gauge generators:

$$
G(t)=P_{\mu} \dot{\xi}^{\mu}+\left(\mathcal{H}_{\mu}+N^{\rho} C_{\mu \rho}^{\nu} P_{\nu}\right) \xi^{\mu}
$$

(where the repeated index, to repeat, involves an integration). Note that $G(t)$ generates variations in the full phase space. It is straightforward to verify that it does generate the correct variations of $N^{\mu}$ (3.8) and (3.9) under the diffeomorphisminduced gauge transformations (3.14).

The preceding discussion applies with no modification of notation to canonical general relativity. 
We continue with some general remarks on diffeomorphism generators. Our first observation is that every generator $G(t)$ with $\xi^{0} \neq 0$ is interpretable as a global time translation generator for at least one member of every gauge equivalence class of solutions. To demonstrate this property we note that for a given set of functions $\xi^{\mu}$ in the expansion

$$
\epsilon^{\mu}=\delta_{a}^{\mu} \xi^{a}+n^{\mu} \xi^{0}
$$

we can solve for the $N^{\mu}$ which render $\epsilon^{\mu}=\delta_{0}^{\mu}$ :

$$
\epsilon^{\mu}=\delta_{0}^{\mu}=\delta_{a}^{\mu} \xi^{a}+\left(N^{-1} \delta_{0}^{\mu}-N^{-1} N^{a} \delta_{a}^{\mu}\right) \xi^{0}
$$

The solution is $N^{\mu}=\xi^{\mu}$, which renders $G(t)$ in Eq. (4.16) identical to the Dirac Hamiltonian (4.13), once we take into account that the equations of motion provide $\dot{N}^{\mu}=\lambda^{\mu}$. Therefore the gauge generator contains, for any solution of the equations of motion, the dynamical evolution as a particular case.

At this point we are ready to understand the coincidence of the two approaches mentioned in the previous section: The same conditions (3.10)-(3.13) are obtained if (1) one asks for the projectability of Eq. (3.7), or (2) one asks for $\left[\delta_{1}, \delta_{2}\right] x^{\mu}$ not to have any explicit time derivative of $\epsilon_{1}$ or $\epsilon_{2}$. It seems odd that conditions imposed on one transformation (projectability) and conditions imposed on the commutation of two transformations should give the same results. The reason lies in the structure of the gauge generators in phase space: They are constructed with linear combinations of constraints with arbitrary functions and their first time derivatives. Let us consider two of these generators $G\left[\xi_{1}\right], G\left[\xi_{2}\right]$. Their Poisson bracket, an equal time commutator, is on general grounds $G\left[\xi_{3}\right]$ for some $\xi_{3}$. It is impossible to get for $\xi_{3}$ the standard diffeomorphism rule $\xi_{3}^{\mu}=\xi_{2}^{\nu} \xi_{1, \nu}^{\mu}-\xi_{1}^{\nu} \xi_{2, \nu}^{\mu}$ : In such a case $\dot{\xi}_{3}$, which appears in $G\left[\xi_{3}\right]$, will depend on the second time derivatives of $\xi_{1}$ and $\xi_{2}$, and this dependence cannot be generated by the equal time Poisson brackets $\left\{G\left[\xi_{1}\right], G\left[\xi_{2}\right]\right\}$. Nesting of Poisson brackets would introduce yet higher time derivatives. This is why general reparametrization covariance cannot be implemented in this form in the Hamiltonian formalism. The argument applies to any reparametrization covariant theory.

This was the argument used in [4] to realize diffeomorphisms in the canonical formalism. In fact the arena in [4] was the reduced space defined by the variables $\left(g_{a b}, K^{a b}\right)$. In this case there are no time derivatives of the arbitrary functions $\xi^{\mu}$ in the variations generated by Eq. (4.16), but the argument still applies in the same way, as shown above. Once the obstruction to projectability is identified through the form of $\left[\delta_{1}, \delta_{2}\right] x^{\mu}$, the assumption of a metric dependence in $\epsilon^{\mu}$ and the requirement that $\left[\delta_{1}, \delta_{2}\right] x^{\mu}$ must not have any explicit time derivative of $\epsilon_{1}$ or $\epsilon_{2}$ leads to equations (3.10)-(3.13), the projectability condition.

We should caution that the algebra (4.3)-(4.5) is satisfied only under the condition that there is no other gauge symmetry in addition to diffeomorphism-induced symmetry. Under more general circumstances, pure diffeomorphisms [even the field dependent variety given by Eq. (3.14)] are not realizable as canonical transformations; they must be accompa- nied by related internal gauge transformations [12]. The issue of projectability for these models will be addressed in another paper.

Gauge theories like electromagnetism or Yang-Mills in Minkowski spacetime share with general relativity the property that gauge transformations (diffeomorphisms in general relativity) need to be constructed with arbitrary functions and their spacetime first derivatives. The gauge generators are made up of two pieces, associated with a primary and a secondary constraint; it is therefore mandatory that all these theories have secondary constraints. This is the way by which the canonical formalism is able to provide us with the right gauge transformations.

For the sake of completeness, let us now apply these ideas to our relativistic particle (3.15). The gauge generator is, from Eq. (4.16),

$$
G(t)=\dot{\xi}(t) \pi+\xi(t) \frac{1}{2}\left(p_{\mu} p^{\mu}+1\right),
$$

with $\xi$ an arbitrary function of time. One can easily check that $G(t)$ generates the (projectable) transformations (3.18) introduced above. Notice also that if $\xi$ is a constant, the secondary constraint generates a rigid (time-independent) Noether symmetry, whereas the primary one does not. Primary constraints generate gauge symmetries only in the case when they do not lead to secondary constraints through the stabilization algorithm.

Finally, notice that we do not modify "by hand" the Hamiltonian by adding to it the secondary constraint with a new Lagrange multiplier. This modification, the so called Dirac conjecture, turns out not only to be unnecessary but to break the equivalence with the Lagrangian theory as well [13].

\section{GAUGE FIXING AND REDUCED FORMALISM}

\section{A. Gauge fixing procedure}

One of the methods to eliminate the superfluous degrees of freedom of a gauge theory is through the introduction of a new set of constraints. This is the gauge fixing procedure, which, according to [5], can be performed in two different steps: the first is to fix the dynamics, the second to fix the redundancy of the initial conditions (though this need not be the order in which the whole set of constraints is introduced).

First, to fix the dynamics-to determine specific values for the functions $\lambda^{\mu}$ in Eq. (4.13) -we must introduce $M+1$ constraints, $\varphi_{\mu} \simeq 0$, such that $\operatorname{det}\left|\left\{\varphi_{\mu}, P_{\nu}\right\}\right| \neq 0$. A typical set could be

$$
\varphi_{\mu}=N^{\mu}-f^{\mu}
$$

with $f^{\mu}\left(f^{0} \neq 0\right)$ a given set of functions not depending on $P_{\mu}$ or $N^{\nu}$ (the simplest choice could be $f^{a}=0, f^{0}=1$ ). We could also think of $f^{\mu}$ as a not yet determined set of functions. These gauge fixing constraints fix $\lambda^{\mu}$ in $H_{\mathrm{D}}$ to be zero and then

$$
H_{\mathrm{D}}^{\mathrm{red}}=N^{\mu} \mathcal{H}_{\mu} \cong f^{\mu} \mathcal{H}_{\mu},
$$

where we have used Dirac's notation of strong equality, $\cong$, to mean an equality up to quadratic pieces in the constraints, 
including the gauge fixing ones. In practice this strong equality tells us that we can substitute $f^{\mu}$ for $N^{\mu}$ within the Hamiltonian due to the fact that $\mathcal{H}_{\mu}$ are constraints, too.

Once this set of evolution-fixing constraints $\varphi_{\mu} \simeq 0$ has been introduced, with a given set of functions $f^{\mu}$, the gauge transformations-strictly speaking-have disappeared. In fact, if we require the gauge generators to be consistent with the new constraints, $\left\{\varphi_{\mu}, G(t)\right\}=0$, we get the relations

$$
\dot{\xi}^{\mu}+\xi^{\nu} N^{\sigma} C_{\nu \sigma}^{\mu}=0,
$$

which means that the functions $\xi^{\mu}$ cease to be arbitrary (at least with respect to the time dependence), and hence there are no more gauge transformations. The transformations $G(t)$ satisfying Eq. (5.3) can be called, as is usual in other contexts, residual gauge transformations, but it must be emphasized that they are not true gauge transformations in phase space, because the arbitrariness that was present in the Dirac Hamiltonian has been eliminated. We encounter a parallel case in electromagnetism, for instance, when after introducing the Lorentz gauge $\partial_{\mu} A^{\mu}=0$, we are left with a residual gauge symmetry, $A^{\mu} \rightarrow A^{\mu}+\partial_{\mu} \Lambda$, provided $\Lambda$ satisfies $\square \Lambda=0$.

Another way to view the residual gauge transformations, which is more interesting to us, is to consider the situation at a given initial time, $t=0$. Let $\alpha^{\mu}(\mathbf{x})=\xi^{\mu}(0, \mathbf{x})$, $\beta^{\mu}(\mathbf{x})=\dot{\xi}^{\mu}(0, \mathbf{x})$; they are related by Eq. (5.3): $\beta^{\mu}+\alpha^{\nu} N^{\sigma} C_{\nu \sigma}^{\mu}=0$. We are left with the "residual" gauge transformation at $t=0$

$$
G_{\mathrm{R}}(0)=P_{\mu} \beta^{\mu}+\left(\mathcal{H}_{\mu}+N^{\rho} C_{\mu \rho}^{\nu} P_{\nu}\right) \alpha^{\mu}=\mathcal{H}_{\mu} \alpha^{\mu},
$$

with $\alpha^{\mu}$ an arbitrary function of $M$-space variables.

The role of $G_{\mathrm{R}}(0)$ is that it generates transformations on the initial value surface that describe a redundancy that is still left in the formalism, and we must eliminate it in order to arrive at the true degrees of freedom. Thus we must introduce a new set of gauge-fixing constraints, $\chi_{\mu} \simeq 0$, with the requirements that (1) the dynamical evolution, which is already fixed, must preserve these new constraints and (2) $\left\{\chi_{\mu}, G_{\mathrm{R}}(0)\right\}=0$ must imply $\alpha^{\mu}=0$.

Obviously, to satisfy the second condition, we need $\operatorname{det}\left|\left\{\chi_{\mu}, \mathcal{H}_{\nu}\right\}\right| \neq 0$, and to satisfy the first we need

$$
\left\{\chi_{\mu}, H_{\mathrm{D}}\right\}+\frac{\partial \chi_{\mu}}{\partial t}=f^{\nu}\left\{\chi_{\mu}, \mathcal{H}_{\nu}\right\}+\frac{\partial \chi_{\mu}}{\partial t} \simeq 0 .
$$

Notice that the first and the second conditions are only compatible if at least one of the gauge fixing constraints $\chi_{\mu}$, for instance $\chi_{0}$, has explicit time dependence: $\partial \chi_{0} / \partial t \neq 0$. This result implies that time needs to be defined classically through a function of the canonical variables.

\section{B. Reduced formalism}

Notice that if we perform the partial gauge fixing defined in Eq. (5.1), $N^{\mu}-f^{\mu} \simeq 0$ in the spirit of keeping $f^{\mu}$ undetermined, then we can interpret

$$
H_{\mathrm{D}}^{\mathrm{red}}=f^{\mu} \mathcal{H}_{\mu}
$$

in Eq. (5.2) as the Hamiltonian for the reduced phase space described by all variables other than $P_{\mu}$ and $N^{\nu}$. In this reduced space we have a dynamical theory defined by a vanishing canonical Hamiltonian and a set of constraints, which now become primary, $\mathcal{H}_{\mu} \simeq 0$. Then the new Dirac Hamiltonian is $H_{\mathrm{D}}^{\text {red }}$ and the new gauge generator is

$$
G^{\mathrm{red}}=\xi^{\mu} \mathcal{H}_{\mu}
$$

Thus we see that the constraints $\mathcal{H}_{\mu}$ do generate gauge transformations in the reduced phase space.

We identify here a frequent source of confusion in the literature when it is claimed that all first class constraints, either primary or secondary (or tertiary, etc.), generate gauge transformations. For generally covariant theories with a metric, in the original phase space, only specific combinations, as in Eq. (4.16), of primary and secondary constraints generate gauge transformations. But in the reduced formalism, since the old secondary constraints take the role of primary constraints and there are no more constraints, these new primary constraints generate gauge transformations in the reduced space.

As to the gauge fixing procedure in the reduced phase space, since there are only primary constraints, there is only one step to be undertaken: to fix the evolution. Notice that the same argument we used previously to show that one of the gauge-fixing constraints must be the definition of time applies here as well.

\section{From the reduced to the original formalism}

In the case of generally covariant theories, we have seen that the reduced formalism consists in the elimination of the primary constraints, $P_{\mu}$, and their canonical conjugate variables, $\quad N^{\mu}$, through a partial gauge fixing $N^{\mu}=f^{\mu}, \quad\left(f^{0} \neq 0\right)$, with $f^{\mu}$ arbitrary functions of spacetime as well as of the reduced variables. Then we obtain a reduced theory which has $\mathcal{H}_{\mu} \simeq 0$ as primary constraints (no secondary constraints appear), $H^{\text {red }}=f^{\mu} \mathcal{H}_{\mu}$ as the Dirac Hamiltonian, and $G^{\text {red }}=\xi^{\mu} \mathcal{H}_{\mu}$ as the generator of gauge symmetries. The new bracket for the set of the reduced variables is just the Dirac bracket, which in our case is trivially obtained as the old Poisson bracket when acting with the reduced variables.

One may wonder whether there is a way to restore the full theory from the reduced one. In these cases where the constraints eliminated in the process of reduction are canonical momenta we will see that there exists such a method. This is the enlargement procedure:

Consider a theory defined by a canonical Hamiltonian $H_{\mathrm{C}}$ and a set of primary constraints $\phi_{\mu} \simeq 0$. The Dirac Hamiltonian is $H_{\mathrm{D}}=H_{\mathrm{C}}+\lambda^{\mu} \phi_{\mu}$ ( $\lambda^{\mu}$ are arbitrary functions). Let us suppose we have applied the stabilization algorithm to obtain secondary, tertiary, etc., constraints and that we have finally obtained a set of Noether gauge generators, described by a single $G[\xi]$, where $\xi$ stands for a set $\xi^{\alpha}$ of infinitesimal arbitrary functions of spacetime. $G[\xi]$ is assumed to be a local functional of $\xi^{\alpha}$ (that is, it depends linearly on $\xi^{\alpha}$ and a finite number of its time derivatives, according to the length of the stabilization algorithm). We also assume the commutation algebra for $G[\xi]$ to be 


$$
\left\{G\left[\xi_{1}\right], G\left[\xi_{2}\right]\right\}=G\left[\xi_{3}\right],
$$

with $\xi_{3}^{\alpha}=C_{\beta \gamma}^{\alpha} \xi_{1}^{\beta} \xi_{2}^{\gamma}$ (this is a general property, and the $C_{\beta \gamma}^{\alpha}$ are not necessarily the same as previously defined; remember that repeated indices imply both summation and integration).

According to Sec. II, there exist functionals $A_{\nu}^{\mu}[\xi]$ and $B^{\mu}[\xi]$ such that

$$
\left\{G[\xi], H_{\mathrm{C}}\right\}+\frac{\partial G[\xi]}{\partial t}=B^{\mu}[\xi] \phi_{\mu}, \quad\left\{G[\xi], \phi_{\nu}\right\}=A_{\nu}^{\mu}[\xi] \phi_{\mu} .
$$

The enlargement procedure consists in promoting the arbitrary functions $\lambda^{\mu}$ to the status of canonical variables; let us call them $N^{\mu}$ for obvious reasons. Let us introduce canonical momenta $P_{\mu}$ associated with the new variables (we thus trivially enlarge the Poisson bracket) and require these new momenta to be the primary constraints of the enlarged theory.

The enlarged canonical Hamiltonian will then be $H_{\mathrm{C}}+N^{\mu} \phi_{\mu}$, and the new Dirac Hamiltonian will be $H_{\mathrm{E}}=H_{\mathrm{C}}+N^{\mu} \phi_{\mu}+\eta^{\mu} P_{\mu}$, with $\eta^{\mu}$ new arbitrary functions. It is straightforward to verify that the dynamics of the original theory and that of the enlarged theory coincide as far as the evolution of the original variables is concerned. However, note that the $\phi^{\mu}$ have become secondary constraints.

Now we will show how to enlarge the corresponding gauge generators $G[\xi]$. Since the new primary constraints $P_{\mu}$ must appear within the enlarged gauge generators $G_{\mathrm{E}}[\xi]$, we will assume the general form $G_{\mathrm{E}}[\xi]$ $=G[\xi]+S^{\mu}[\xi] P_{\mu}$, with $S^{\mu}$ to be determined through the requirements of Sec. II. It turns out that $S^{\mu}$ $=B^{\mu}+N^{\nu} A_{\nu}^{\mu} P_{\mu}$. The enlarged gauge generator therefore has the following form:

$$
G_{\mathrm{E}}[\xi]=G[\xi]+B^{\mu}[\xi] P_{\mu}+N^{\nu} A_{\nu}^{\mu}[\xi] P_{\mu} .
$$

The commutation algebra for $G_{\mathrm{E}}[\xi]$ is

$$
\left\{G_{\mathrm{E}}\left[\xi_{1}\right], G_{\mathrm{E}}\left[\xi_{2}\right]\right\}=G_{\mathrm{E}}\left[\xi_{3}\right]+\mathcal{O}\left(P^{2}\right),
$$

where $\mathcal{O}$ is pure quadratic in the (new) primary constraints:

$$
\mathcal{O}\left(P^{2}\right)=\left\{B^{\mu}\left[\xi_{1}\right]+N^{\nu} A_{\nu}^{\mu}\left[\xi_{1}\right], B^{\sigma}\left[\xi_{2}\right]+N^{\rho} A_{\rho}^{\sigma}\left[\xi_{2}\right]\right\} P_{\mu} P_{\sigma} .
$$

In our particular case of general covariant theories, $B^{\mu}[\xi]=\dot{\xi}^{\mu}$ and $A_{\nu}^{\mu}[\xi]=\xi^{\rho} C_{\rho \nu}^{\mu}$. Notice that in this particular case the term $\mathcal{O}\left(P^{2}\right)$ vanishes.

The procedure of enlargement here devised is completely general, and it is valid for any gauge theory no matter how complicated its structure of constraints may be.

\section{GAUGE GROUP}

The gauge group is a subgroup of the symmetry group of the system. A symmetry is a transformation that maps solutions of the equations of motion into solutions. From a physical standpoint, gauge symmetry reflects a redundancy in the description. Mathematically, a gauge transformation is characterized by its functional dependence on arbitrary functions. The functional dependence is expected to be local in the sense of depending on the values of the functions and on a finite number of derivatives. This is the definition for classi- cal mechanics and classical field theory. It is most convenient to define gauge symmetries in a more restrictive way as local transformations which leave the action invariant up to boundary terms. Our analysis is based on the Noether identities which result from this invariance under infinitesimal local symmetries.

Let us make some formal remarks on the nature of the diffeomorphism-induced gauge group of the type discussed in this paper. Let $\operatorname{Riem}(\mathcal{M})$ be the space of (pseudo) Riemannian metrics of the spacetime manifold $\mathcal{M}$, and let $\operatorname{Diff}(\mathcal{M})$ be the group of diffeomorphisms in $\mathcal{M}$. An element of the gauge group $\mathbf{G}[\operatorname{Riem}(\mathcal{M})]$ is a regular map $\operatorname{Riem}(\mathcal{M}) \rightarrow \operatorname{Riem}(\mathcal{M})$ such that each $\mathrm{g} \in \operatorname{Riem}(\mathcal{M})$ undergoes a diffeomorphic transformation, that is, a transformation dictated by a specific element of $\operatorname{Diff}(\mathcal{M})$ (thus keeping the action invariant). (Other fields are also affected by this diffeomorphic transformation, but for this discussion we devote our attention to the metric.) But this element of $\operatorname{Diff}(\mathcal{M})$ may be different if we consider the action of the same element of the gauge group on a different $g^{\prime}$ $\in \operatorname{Riem}(\mathcal{M})$.

To determine an element of the gauge group we must assign to each $g \in \operatorname{Riem}(\mathcal{M})$ the specific spacetime diffeomorphism which is going to act on $g$. More precisely, an element, $d$, of the gauge group is a map

$$
\begin{gathered}
d: \operatorname{Riem}(\mathcal{M}) \rightarrow \operatorname{Diff}(\mathcal{M}) \\
g \rightarrow d[g]
\end{gathered}
$$

such that we can build out of it a regular map

$$
\begin{gathered}
\mathbf{G :} \operatorname{Riem}(\mathcal{M}) \rightarrow \operatorname{Riem}(\mathcal{M}) \\
g \rightarrow(d[g])(g) .
\end{gathered}
$$

Now let us consider the generators of the gauge group $\mathbf{G}$. We use, for the sake of generality, a condensed notation where $\Phi^{i}$ stands for the fields that are present in the theory; the action is denoted by $\mathcal{S}$, and $i$ includes continuous spacetime indices (so that repeated indices imply both summation and integration). Let $\epsilon^{\alpha}$ be arbitrary functions of spacetime variables, and $\delta_{\epsilon} \Phi^{i}=R_{\alpha}^{i} \epsilon^{\alpha}$ be a complete set of infinitesimal gauge transformations. These satisfy the Noether identities $\left(\delta \mathcal{S} / \delta \Phi^{i}\right) R_{\alpha}^{i}=0$. Obviously we do not alter the Noether identities by taking a different linear combination of variations $R_{\alpha}^{i} \rightarrow \bar{R}_{\alpha}^{i}=\Lambda_{\alpha}^{\beta}(\Phi) R_{\beta}^{i}$, even when the $\Lambda_{\alpha}^{\beta}$ depend on $\Phi^{i}$. No gauge equivalent trajectories are eliminated through this transformation, presuming that $\boldsymbol{\Lambda}$ is invertible. In our case [see Eq. (3.14)] the requirement of projectability fixed

$$
\Lambda_{\nu}^{\mu}(g)=n^{\mu} \delta_{\nu}^{0}+\delta_{a}^{\mu} \delta_{\nu}^{a},
$$

which is clearly invertible. The algebra corresponding to this new choice of generators contains field-dependent structure coefficients.

One might conclude that this "soft" algebra structure signifies that the symmetry transformations no longer form a group. We do have a group, which acts not on the spacetime manifold $\mathcal{M}$ but on $\operatorname{Riem}(\mathcal{M})$. Note that elements of the diffeomorphism-induced gauge group must depend on the full metric. Dependence on the lapse and shift is fixed by Eq. 
(3.14). Perhaps more surprising is the fact that the full group depends nonlocally on the hypersurface induced metric. This is a direct consequence of the structure coefficients in Eq. (4.6): Repeated nesting of the commutator produces spatial derivatives of $g_{a b}$ to infinite order [1].

To summarize, we have discussed some aspects of the canonical approach to generally covariant theories. In particular we have emphasized the special way in which the canonical formalism describes the diffeomorphism covariance of these theories. The gauge group for these theories is larger than the diffeomorphism group. The canonical gauge generators are just one of the possible bases for the gauge algebra, although projectability of transformations generated by the larger group from configuration-velocity space to phase space fixes the dependence on the lapse and shift uniquely. We have displayed the canonical generators for the gauge symmetries of these theories on the entire phase space. Transformations may be pulled back to the entire configuration-velocity space.

In this paper we assumed that the only gauge symmetries are generated by diffeomorphisms. When other gauge symmetries occur, related internal gauge transformations must be taken into account. This topic and the question of projectability of the gauge transformation group onto the full constraint hypersurface will be dealt with in future papers. It is also our intention to explore further the relationship between our results and matters pertaining to quantization, particularly the question of time in quantum gravity.

\section{ACKNOWLEDGMENTS}

J.M.P. and D.C.S. would like to thank the Center for Relativity of The University of Texas at Austin for its hospitality. J.M.P. acknowledges support by CICYT Contracts No. AEN95-0590 and No. GRQ 93-1047. D.C.S. acknowledges support by National Science Foundation Grant No. PHY94-13063.

\section{APPENDIX: NAMBU-GOTO RELATIVISTIC STRING}

We have so far discussed the situation in which the metric may be constructed using only configuration space variables. Our conclusions, however, do hold in case that velocity variables, too, are needed to construct the metric. In this appendix we illustrate the issue of projectability of variations engendered by diffeomorphisms in the Nambu-Goto relativistic string. This is an example of a first order dynamical model in which a metric may be constructed using velocity as well as configuration variables. (The Polyakov string satisfies the conditions postulated in Sec. III.)

We let $\left\{y^{I}, I=0, \ldots, M\right\}$ represent Minkowski spacetime coordinates. The string surface is given by $y^{I}\left(x^{\mu}\right), \quad \mu=0,1$, where $x^{0}=\tau$ and $x^{1}=\sigma$. The induced metric on the string surface is

$$
g_{\mu \nu}=y_{, \mu}^{I} y_{, \nu}^{J} \eta_{I J} .
$$

For spacetime contractions we use the notation $\left(\cdot=d / d \tau,{ }^{\prime}\right.$ $=d / d \sigma)$

$$
\left(\dot{y}^{2}\right)=\dot{y}^{I} \dot{y}_{I}, \quad\left(y^{\prime 2}\right)=y^{\prime} y_{I}^{\prime}, \quad\left(\dot{y} y^{\prime}\right)=\dot{y}^{I} y_{I}^{\prime} .
$$

The Lagrangian density is minus the string volume element :

$$
L=-(-\operatorname{det} g)^{1 / 2}=-\left[-\left(\dot{y}^{2}\right)\left(y^{\prime 2}\right)+\left(\dot{y} y^{\prime}\right)^{2}\right]^{1 / 2} .
$$

From

$$
g^{\mu \nu}=-L^{-2}\left(\begin{array}{cc}
\left(y^{\prime 2}\right) & -\left(\dot{y} y^{\prime}\right) \\
-\left(\dot{y} y^{\prime}\right) & \left(\dot{y}^{2}\right)
\end{array}\right),
$$

we read off the lapse and shift:

$$
N=\frac{L}{\left(y^{\prime 2}\right)^{1 / 2}}
$$

and

$$
N^{1}=\frac{\left(\dot{y} y^{\prime}\right)}{\left(y^{\prime 2}\right)}
$$

The canonical momentum is

$$
\hat{\pi}^{I}=\frac{\partial L}{\partial \dot{y}_{I}}=-L^{-1}\left[\left(y^{\prime}\right)^{2} \dot{y}^{I}-\left(\dot{y} y^{\prime}\right) y^{\prime I}\right]
$$

Recall that when we are working in configuration-velocity space, the coordinates are $\left\{y^{I}, \dot{y}^{I}\right\}$. There are two primary constraints in phase space:

$$
\phi_{0}=\frac{1}{2}\left[(\pi)^{2}+\left(y^{\prime}\right)^{2}\right]
$$

and

$$
\phi_{1}=\left(y^{\prime} \pi\right)
$$

Therefore one may ask whether the velocities may be expressed uniquely in terms of the canonical momenta and the lapse and shift, taking into account that these constraints show that there are a correct number of coordinates $\left\{y^{I}, \hat{\pi}^{I}, N, N^{1}\right\}$ for velocity space. In our example we can indeed invert the expression for $\hat{\pi}^{i}$ to obtain

$$
\dot{y}^{I}=N^{1} y^{\prime I}-\frac{N}{\left(y^{\prime 2}\right)^{1 / 2}} \hat{\pi}^{I} .
$$

The primary constraints are relations among the $y^{I}$ and the $\pi^{I}$ and involve neither lapse nor shift. Therefore invertibility is equivalent to the demand that

$$
0=\frac{\partial \hat{\pi}_{I}}{\partial N^{\mu}}=\frac{\partial \hat{\pi}_{I}}{\partial \dot{y}^{J}} \frac{\partial \dot{y}^{J}}{\partial N^{\mu}}=W_{I J} \frac{\partial \dot{y}^{J}}{\partial N^{\mu}},
$$

where 


$$
\begin{aligned}
W_{I J}= & \frac{\partial^{2} L}{\partial \dot{y}^{I} \partial \dot{y}^{J}} \\
= & -\frac{1}{L^{3}}\left[L^{2}\left(y^{\prime 2}\right) \delta_{I J}+\left(\dot{y}^{2}\right)\left(y^{\prime 2}\right) y_{I}^{\prime} y_{J}^{\prime}+\left(y^{\prime 2}\right)^{2} \dot{y}_{I} \dot{y}_{J}\right. \\
& \left.-\left(\dot{y} y^{\prime}\right)\left(y^{\prime 2}\right)\left(\dot{y}_{I} y_{J}^{\prime}+y_{I}^{\prime} \dot{y}_{J}\right)\right] \\
= & -\frac{1}{N\left(y^{\prime 2}\right)^{1 / 2}}\left[\left(y^{\prime 2}\right) \delta_{I J}-y_{I}^{\prime} y_{J}^{\prime}+\hat{\pi}_{I} \hat{\pi}_{J}\right] .
\end{aligned}
$$

But this is the statement that

$$
\frac{\partial \dot{y}^{I}}{\partial N}=-\frac{1}{\left(y^{\prime 2}\right)^{1 / 2}} \hat{\pi}^{I} \text { and } \frac{\partial \dot{y}^{I}}{\partial N^{1}}=y^{\prime I}
$$

are null eigenvectors of $W_{I J}$; that they are is readily verified.

Recall that in our model the primary constraints are $\phi_{0}=\frac{1}{2}\left[\left(y^{\prime}\right)^{2}+\left(\pi^{2}\right)\right]$ and $\phi_{1}=\left(y^{\prime} \pi\right)$. We have

$$
\gamma_{0}^{I}=\mathcal{F} L^{*}\left(\frac{\partial \phi_{0}}{\partial \pi_{I}}\right)=\hat{\pi}^{I} \text { and } \gamma_{1}^{I}=\mathcal{F} L^{*}\left(\frac{\partial \phi_{1}}{\partial \pi_{I}}\right)=y^{\prime I}
$$

Thus

$$
\boldsymbol{\Gamma}_{0}=\hat{\pi}^{I} \frac{\partial}{\partial \dot{y}^{I}}=-\left(y^{\prime 2}\right)^{1 / 2} \frac{\partial}{\partial N} \quad \text { and } \quad \boldsymbol{\Gamma}_{1}=y^{\prime I} \frac{\partial}{\partial \dot{y}^{I}}=\frac{\partial}{\partial N^{1}} .
$$

Therefore variations of the $y^{I}$ will be projectable if and only if they are independent of $N$ and $N^{1}$. These variations are

$$
\delta y^{I}=y_{, \mu}^{I} \epsilon^{\mu}=\dot{y}^{I} \epsilon^{0}+y^{\prime I} \epsilon^{1}=\left(N^{1} y^{\prime I}-\frac{N}{\left(y^{\prime 2}\right)^{1 / 2}} \hat{\pi}^{I}\right) \epsilon^{0}+y^{\prime I} \epsilon^{1} .
$$

It is straightforward to show that these variations will be independent of $N, N^{1}$ if and only if

$$
\epsilon^{0}=\frac{1}{N} \xi^{0}, \quad \epsilon^{1}=-\frac{N^{1}}{N} \xi^{0}+\xi^{1}
$$

where $\xi^{\mu}$ are independent of $N, N^{1}$. In terms of the timelike unit vector

$$
n^{\mu}=\left(1 / N,-N^{1} / N\right)
$$

this result is in the correct form, namely the same as Eq. (3.13):

$$
\epsilon^{\mu}=\delta_{1}^{\mu} \xi^{1}+n^{\mu} \xi^{0}
$$

[1] P. G. Bergmann and A. Komar, Int. J. Theor. Phys. 5, 15 (1972).

[2] C. J. Isham and K. V. Kuchař, Ann. Phys. (N.Y.) 164, 288 (1985); 164, 316 (1985).

[3] J. Lee and R. M. Wald, J. Math. Phys. (N.Y.) 31, 725 (1990).

[4] D. C. Salisbury and K. Sundermeyer, Phys. Rev. D 27, 740 (1983).

[5] J. M. Pons and L. C. Shepley, Class. Quantum Grav. 12, 1771 (1995).

[6] C. Batlle, J. Gomis, X. Gràcia, and J. M. Pons, J. Math. Phys. (N.Y.) 30, 1345 (1989).

[7] K. Kamimura, Nuovo Cimento B 68, 33 (1982).
[8] C. Batlle, J. Gomis, J. M. Pons, and N. Roman-Roy, J. Math. Phys. (N.Y.) 27, 2953 (1986).

[9] X. Gràcia, J. M. Pons, and J. Roca, Int. J. Mod. Phys. A 9, 5001 (1994).

[10] R. M. Wald, General Relativity (University of Chicago Press, Chicago, 1984).

[11] C. Batlle, J. Gomis, J. París, and J. Roca, Phys. Lett. B 224, 228 (1989).

[12] D. C. Salisbury and K. Sundermeyer, Phys. Rev. D 27, 757 (1983).

[13] X. Gràcia and J. M. Pons, J. Phys. A 21, 2705 (1988). 